\title{
EDITORIAL
}

\section{Evitemos la inseguridad alimentaria en tiempos de COVID-19 en Chile}

\section{Let's avoid food insecurity in COVID-19 time in Chile}

La pandemia por COVID-19 no ha dejado ningún ámbito de nuestras vidas sin afectar. La alimentación y nutrición de individuos y de la población se han visto forzosamente afectados; ha cambiado la distribución, disponibilidad y acceso a los alimentos y, posiblemente, inclusive su forma de producción. De acuerdo a la Organización de las Naciones Unidas para la Alimentación y la Agricultura (FAO), "la mayoría de las evaluaciones actuales prevén una contracción en la oferta y la demanda de productos agrícolas, señalando posibles interrupciones en el comercio y la logística" (p.2)

Podemos suponer que la pandemia ha modificado nuestros ambientes y hábitos alimentarios, incluyendo cambios en la forma en que adquirimos los alimentos, en su preparación y en el consumo. En este sentido, la alimentación y la nutrición se podrían ver perjudicadas por el confinamiento, la disminución del poder adquisitivo familiar, por la depresión económica y por una potencial falta de disponibilidad y dificultad de acceso a alimentos sanos y seguros, en especial en los grupos más vulnerables ${ }^{2}$.

Para algunos grupos privilegiados, estar en cuarentena puede significar disponer de más tiempo para hacer ejercicios y cocinar en casa en base a productos naturales, legumbres, pescados, frutas y verduras, facilitados por el despacho a domicilio, y una menor exposición a ambientes obesogénicos, constituyéndose incluso en una oportunidad de tener un estilo de vida más saludable. Sin embargo, para la gran mayoría de la población, la cuarentena se traduce en hacinamiento, empobrecimiento, sedentarismo, violencia intrafamiliar, sobrecarga de tareas domésticas para las mujeres ${ }^{3,4}$, menor acceso a alimentos saludables, y dificultades de acceso a los programas estatales de alimentación complementaria de salud y de alimentación escolar. Estos hechos generan inseguridad alimentaria en términos de menor cantidad de alimentos disponibles y de baja calidad nutricional (alto contenido calórico, de azúcares, sodio y grasas saturadas), muy por el contrario de lo que se ha planteado respecto a la importancia de acceder a una alimentación balanceada como una de las medidas para evitar complicaciones graves por COVID-19 $9^{1,2}$.

El contexto actual se vislumbra adverso y probablemente se exacerben las desigualdades alimentarias y nutricionales en nuestro país, y aumente la obesidad, condición que además se ha descrito como de mayor riesgo para la severidad de la enfermedad por coronavirus ${ }^{5,6}$.

El Director General de FAO, Qu Dongyu ha planteado que para reducir el riesgo de un costo aún mayor (escasez de alimentos para millones, incluso en países ricos), "el mundo debe tomar medidas inmediatas para minimizar las alteraciones de las cadenas de suministro de alimentos. La incertidumbre sobre la disponibilidad de alimentos puede llevar a los encargados de formular políticas a implementar medidas comerciales restrictivas para salvaguardar la seguridad alimentaria nacional"7. En momentos de crisis, nos vemos obligados a problematizar el sistema alimentario global y a fortalecer los sistemas regionales de tal manera que sean más sostenibles, sustentables y saludables, fomentando la producción y el abastecimiento local. Se hace presente la necesidad de colaboración entre países, pero también el fortalecimiento de los vínculos entre diversos sectores y actores del canal agroalimentario, para mitigar -en lo inmediato- las consecuencias de cadenas alimentarias deficientes que afectan a las poblaciones más vulnerables, pero que a largo plazo involucran a toda la población $n^{8,9}$.

Chile, en gran parte de sus alimentos básicos (entre ellos trigo, arroz, legumbres) no es autosuficiente. Por ejemplo, en trigo debemos importar un porcentaje que fluctúa entre el $30 \%$ y $40 \%$; en arroz ese valor es en promedio un 50\%; en legumbres, en el caso de lenteja, casi el 90\% es importado, situación similar a la del garbanzo y en menor proporción el poroto. La mayor parte de los terrenos históricamente dedicados a cultivos de legumbres, han sido desplazados por cultivos más rentables para la exportación como frutales, disminuyendo fuertemente la superficie de siembra. A esto se suma el problema de estrés hídrico, lo que complejiza el escenario 
de autoabastecimiento.

Preocupados por la inseguridad alimentaria de la población chilena en tiempos de pandemia por COVID-19, proponemos:

- Retomar la discusión sobre el derecho constitucional a la alimentación inocua y saludable que renació durante el estallido social para asegurar entornos alimentarios seguros y saludables, que resistan las condiciones de crisis.

- Vigilancia y, si es necesario, regulación estatal de precios de los alimentos.

- Garantía de acceso físico y económico a frutas, verduras, legumbres y pescados a través de subsidios a personas/familias vulnerables.

- Difusión de un mensaje de equidad, orientado a incentivar la distribución familiar de las tareas de alimentación y cuidado, para evitar la sobrecarga de las mujeres.

- Reforzar los programas de entrega estatal de alimentos (PNAC, PACAM, PAE), asegurando que los beneficiarios accedan a sus alimentos (entrega en casa y aumentando transitoriamente las entregas para satisfacer más necesidades).

- Facilitar los circuitos cortos de comercialización de alimentos resolviendo problemas logísticos de distribución y almacenaje.

- Apoyo económico y sanitario a feriantes, pequeños productores agrícolas y pesqueros.

- Garantizar la operación de puntos de venta de alimentos saludables, especialmente al por menor y mercados locales. (por ejemplo, redistribución de puntos de feria en toda la comunidad).

- Otras medidas que mantengan el intercambio comercial para evitar alteraciones en los precios y déficit de insumos.

Creemos que el Estado debe velar por el bienestar de la población, por el derecho a la alimentación y por el acceso y disponibilidad de alimentos básicos, inocuos y saludables para todos y todas ${ }^{8,10}$.

\section{BIBLIOGRAFÍA}

1. Organizacion de las Naciones Unidas para la Alimentacion y la Agricultura. Análisis y respuestas de América Latina y el Caribe ante los efectos de COVID-19 en los sistemas alimentarios. 2020. Disponible en: http:// www.fao.org/americas/publicaciones-audio-video/covid19-y-sistemas-alimentarios/es/

2. Butler MI, Barrientos RM. The impact of nutrition on COVID-19 susceptibility and long-term consequences. Brain Behav Immun. 2020; Disponible en: https://www.ncbi.nlm.nih.gov/pmc/articles/PMC7127800/pdf/ main.pdf

3. Wenham C, Smith J, Morgan R, Gender and COVID-19 Working Group. COVID-19: the gendered impacts of the outbreak. Lancet. 2020; 10227: 846-848.

4. United Nations Population Fund. Informe Tecnico Covid-19: Un enfoque de género proteger la salud y los derechos sexuales y reproductivos y promover la igualdad de género. 2020. Disponible en: https://www. unfpa.org/sites/default/files/resource-pdf/COVID-19_A_Gender_Lens_Guidance_Note.docx_en-US_es-MX. pdf

5. Qingxian C, Fengjuan C, Fang L, Xiaohui L, Tao W, Qikai W, et al. Obesity and COVID-19 Severity in a Designated Hospital in Shenzhen, China. Lancet Infect Dis. 2020; (202002073000001).

6. Ministerio de Salud de Chile. Encuesta Nacional de Salud 2016-2017. Segunda entrega de resultados. 2018. Disponible en: https://www.minsal.cl/wp-content/uploads/2018/01/2-Resultados-ENS_MINSAL_31_01_2018. pdf

7. Organización de las Naciones Unidas para la Alimentación y la Agricultura. Es crucial mantener las cadenas alimentarias mundiales en la crisis COVID-19. 2020. Disponible en: http://www.fao.org/nicaragua/noticias/ detail-events/en/c/1268844/

8. Naja F, Hamadeh R. Nutrition amid the COVID-19 pandemic: a multi-level framework for action. Eur I Clin Nutr. 2020; 1-5. Disponible en: http://www.nature.com/articles/s41430-020-0634-3

9. Organizacion de las Naciones Unidas para la Alimentacion y la Agricultura, Comunidad de Estados 
Latinoamericanos y Caribenos. Seguridad Alimentaria bajo la Pandemia de COVID-19. 2020. Disponible en: https://www.alainet.org/es/articulo/206214

10. GTOP. El Derecho Constitucional a la Alimentación [Internet]. 2019. Available from: https://www.uchile.cl/ noticias/159046/el-derecho-constitucional-a-la-alimentacion

Lorena Rodríguez Osiac ${ }^{*}$, Daniel Egaña Rojas², Patricia Gálvez Espinoza ${ }^{3}$, Deborah Navarro-Rosenblatt ${ }^{4}$, Marcela Araya $B^{5}$, María Begoña Carroza 5 , Cecilia Baginsky $G^{6}$.

En nombre del Grupo Transdisciplinario de Obesidad de Poblaciones, GTOP, Universidad de Chile.

1. Escuela de Salud Pública Dr. Salvador Allende G., Universidad de Chile.

2. Departamento de Atención Primaria y Salud Familiar, Facultad de Medicina, Universidad de Chile.

3. Departamento de Nutrición, Facultad de Medicina, Universidad de Chile.

4. Programa de Doctorado, Escuela de Salud Pública Dr. Salvador Allende G., Universidad de Chile.

5. Departamento de Promoción de la Salud de la Mujer y el Recién Nacido, Facultad de Medicina, Universidad de Chile.

6. Académica, Facultad de Ciencias Agronómicas, Universidad de Chile

*Autor de correspondencia: Dra. Lorena Rodríguez Osiac Escuela de Salud Pública Dr. Salvador Allende G., Universidad de Chile. Dirección: Av. Independencia 939, Santiago, Chile Teléfono: +569 98883559 Email: Irodriguezosiac@gmail.com 